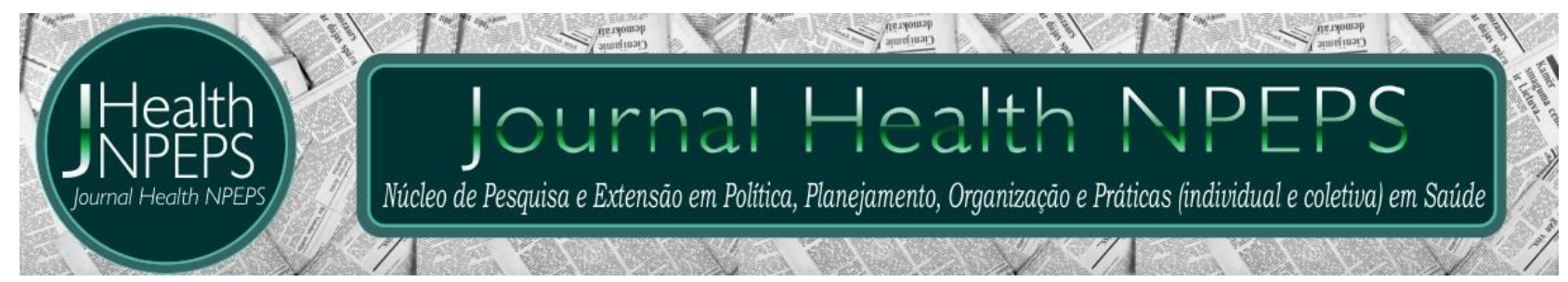

http://dx.doi.org/10.30681/252610103129

ARTIGO ORIGINAL

\title{
Validación de un inventario de conductas sexuales en hombres que tienen sexo con hombres
}

\section{Validation of an inventory of sexual behaviors in men who have sex with men}

\section{Validação de um inventário de comportamentos sexuais em homens que fazem sexo com homens}

\section{Carolina Valdez-Montero ${ }^{1}$, José Moral de la Rubia ${ }^{2}$, Dora Julia Onofre-Rodríguez ${ }^{3}$, Raquel Alicia Benavides-Torres ${ }^{4}$, Jesica Guadalupe Ahumada-Cortez ${ }^{5}$}

\begin{abstract}
RESUMEN
Objetivo: determinar la validez del cuestionario de conductas sexuales en hombres que tienen sexo con hombres (HSH). Método: se realizó una investigación instrumental con un diseño ex post-facto transversal. Se usó un muestreo dirigido por entrevistados para reclutar a $133 \mathrm{HSH}$ mexicanos. Resultados: los ocho ítems de frecuencia de relaciones sexuales definieron dos escalas independientes: sexo con mujeres ( $a$ ordinal $=.926$ ) y sexo con hombres (a ordinal $=.898$ ). Ambos modelos unidimensionales mostraron ajustes aceptables. La escala de sexo desprotegido tuvo una estructura de tres factores con ajuste
\end{abstract}

\footnotetext{
${ }^{1}$ Doctora en Ciencias de Enfermería. Profesora-investigadora de Tiempo Completo, miembro del Sistema Nacional de Investigadores Nivel 1. Universidad Autónoma de Sinaloa, Escuela Superior de Enfermería. Mochis, Sinaloa, México. Email: carolina.valdez@uas.edu.mx ORCID ID: https://orcid.org/0000-0002-4938-3087 Autor principal - Dirección de envío: Fuente de Poseidón y Ángel Flores s/n, Los Mochis, Ahome, Sinaloa, México.

${ }^{2}$ Doctor en Filosofía y Ciencias de la Educación. Profesor-investigador de Tiempo Completo. Miembro del Sistema Nacional de Investigadores Nivel 2. Universidad Autónoma de Nuevo León. Facultad de Psicología. Monterrey, Nuevo León, México. E-mail: jose moral@hotmail.com ORCID ID: http://orcid.org/0000-0003-1856-1458

${ }^{3}$ Posdoctorado y Doctora en Filosofía con Orientación en Trabajo Social y Políticas Comparadas de Bienestar Social. Profesora-investigadora de Tiempo Completo. Miembro del Sistema Nacional de Investigadores Nivel 1. Universidad Autónoma de Nuevo León, Facultad de Enfermería. CIDICS, Monterrey, Nuevo León, México. E-mail: donofre64@yahoo.com.mx ORCID ID: https://orcid.org/0000-0003-1214-9761 Correspondencia: Pedro de Alba s/h, San Nicolás de Los Garza, Nuevo León, México.

${ }^{4} \mathrm{PhD}$. Profesora-investigadora de Tiempo Completo. Miembro del Sistema Nacional de Investigadores Nivel 1. Universidad Autónoma de Nuevo León, Facultad de Enfermería. Monterrey, Nuevo León, México. E-mail: rabenavi@gmail.com ORCID ID: https://orcid.org/0000-0001-5113-4250

${ }^{5}$ Doctora en Ciencias de Enfermería. Profesora Investigadora de Tiempo Completo. Universidad Autónoma de Sinaloa, Escuela Superior de Enfermería. Mochis, Sinaloa, México. E-mail: jesica.ahumada.cortez@uas.edu.mx ORCID ID: http://orcid.org/0000-0003-0092-0221
} desde que a publicação original seja corretamente citada.
} 
Valdez-Montero C, Rubia JM, Onofre-Rodríguez DJ, Benavides-Torres RA, Ahumada-Cortez JG. Validación...

aceptable: con trabajadores/as sexuales (a ordinal $=.961)$, con parejas ocasionales ( $a$ ordinal $=.849$ ) y con pareja estable (a ordinal $=.858$ ). Conclusión: el cuestionario muestra consistencia interna y validez estructural.

Descriptores: Sexualidad; Salud del hombre; Homosexualidad; Conductas de riesgo; Psicometría.

ABSTRACT

Objective: to determine the validity of the sexual behavior questionnaire in men who have sex with men (MSM). Method: an instrumental research was carried out with an expost-facto cross-sectional design. Respondent driven sampling was used to recruit 133 MSM Mexican. Results: the eight items of frequency of sexual relations defined two independent scales: sex with women (a ordinal $=.926$ ) and sex with men ( $a$ ordinal $=.898$ ). Both one-dimensional models showed acceptable adjustments. The unprotected sex scale had a structure of three factors with acceptable adjustment: with sex workers ( $a$ ordinal = .961 ), with occasional partners ( $a$ ordinal $=.849$ ) and with a stable partner ( $a$ ordinal $=$ .858). Conclusion: the questionnaire shows internal consistency and structural validity.

Descriptors: Sexuality; Men's health; Homosexuality; Risk behaviors; Psychometry.

\section{RESUMO}

Objetivo: determinar a validade do questionário de comportamento sexual de homens que fazem sexo com homens (HSH). Método: uma investigação instrumental foi realizada com um design ex-post-facto de seção transversal. Uma amostra dirigida por entrevistados foi usada para recrutar $133 \mathrm{HSH}$ mexicanos. Resultados: os oito itens de frequência de relações sexuais definiram duas escalas independentes: sexo com mulheres $(a$ ordinal $=.926)$ e sexo com homens (a ordinal $=0,898$ ). Ambos os modelos unidimensionais mostraram ajustes aceitáveis. A escala sexual desprotegida tinha uma estrutura de três fatores com ajustamento aceitável: com profissionais do sexo (a ordinal $=0,961)$, com parceiros ocasionais (a ordinal $=0,849$ ) e com um parceiro estável (a ordinal $=0,858$ ). Conclusão: o questionário mostra consistência interna e validade estrutural.

Descritores: Sexualidade; Saúde do homem; Homossexualidade; Comportamentos de risco; Psicometria.

\section{INTRODUCCIÓN}

De acuerdo a la Organización Mundial de la Salud ${ }^{1}$, el Virus de Inmunodeficiencia Humana (VIH) continúa siendo uno de los agentes infecciosos más mortales en el mundo, debido a que ha cobrado más de 35 millones de vidas. Según el Centers for Disease Control and Prevention ${ }^{2}$, el $\mathrm{VIH}$ se transmite principalmente por relaciones sexuales orales, vaginales y anales sin uso del condón, siendo el coito anal el tipo de sexo más riesgoso. El coito anal se presenta mayormente en hombres que tienen sexo con hombres (HSH), este término es utilizado para describir a los hombres que tienen prácticas sexuales con otros 
Valdez-Montero C, Rubia JM, Onofre-Rodríguez DJ, Benavides-Torres RA, Ahumada-Cortez JG. Validación...

hombres sin importar su identidad u orientación sexual ${ }^{3}$. De acuerdo a la OMS ${ }^{4}$, los HSH tienen casi 20 veces más probabilidades de infectarse del VIH que el resto de la población. Asimismo, las relaciones sexuales con parejas casuales o con trabajadoras (res) sexuales y sin uso del condón son frecuentes en esta población, lo que se considera mayor vulnerabilidad para adquirir del $\mathrm{VIH}^{5}$.

Diversas investigaciones se han enfocado a estudiar la conducta sexual en HSH sin utilizar instrumentos validados o incluyen una gama incompleta de conductas sexuales relevantes para infecciones de transmisión sexual, ${ }^{6,7}$. Por ejemplo, el estudio de Fendrich, Smith, Pollack y Mackesy-Amiti, evaluaron la conducta sexual en HSH en la ciudad de San Francisco en Estados Unidos, considerando el sexo oral, anal y el uso del condón ${ }^{8}$. A su vez, Li, Liu, Liu, Feng y Cai diseñaron una escala de conducta sexual de riesgo en HSH de Shenzhen en China. En este estudio, se incluyeron las relaciones sexuales con parejas regulares y trabajadoras sexuales; sin embargo, la escala solamente medía las relaciones sexuales orales y anales, no se consideró el sexo vaginal, a pesar de que podrían tener sexo con mujeres ${ }^{9}$. Otros autores como Newcomb, Ryan, Garofalo y Mustanski remarcan que no sólo es necesario conocer los tipos de relaciones sexuales (orales, anales y vaginales), sino también los diferentes tipos de parejas sexuales (estable, casuales y trabajadores/as sexuales) en los $\mathrm{HSH}^{10}$.

Asimismo, los instrumentos de conducta sexual se enfocan en medidas dicotómicas, reducido a dos categorías, teniendo como desventaja pérdida de información cuantitativa y el riesgo de infección de adquirir el VIH, por lo que no debería estar reducido a solo dos categorías $^{7}$. A su vez, estudios que han medido la conducta sexual en HSH carecen de instrumentos confiables o validados ${ }^{6,7}$. Debido a estas limitantes en los instrumentos, existe la necesidad de desarrollar un cuestionario con adecuadas propiedades psicométricas.

Considerando este antecedente, se ha diseñado un cuestionario para evaluar la conducta sexual de HSH integrado por una pregunta con formato de respuesta cerrada sobre el número de parejas sexuales y por dos escalas tipo Likert. Una escala evalúa frecuencia de conductas sexuales con mujeres y con hombres; y otra escala, frecuencia de los diferentes tipos de sexo con uso del condón y los tipos de parejas (estable, casuales y trabajadores/as sexuales ${ }^{11}$. El concepto de conducta sexual en HSH evaluado, se definió Journal Health NPEPS. 2018 jul-dez; 3(2):327-351. 
Valdez-Montero C, Rubia JM, Onofre-Rodríguez DJ, Benavides-Torres RA, Ahumada-Cortez JG. Validación...

como el número de parejas sexuales y la frecuencia con la que se tiene sexo vaginal con mujeres, sexo oral activo y pasivo y sexo anal insertivo con mujeres y hombres y sexo anal receptivo con hombres. En este concepto se incluyó la frecuencia del uso de condón o sexo protegido al mantener en estas conductas sexuales, considerando tres tipos de pareja, ya sea masculina o femenina: pareja estable (persona con la que se tiene una relación de noviazgo, convivencia o matrimonio), pareja casual (persona con la que mantienen relaciones sexuales mutuamente consentidas sin mediar un pago económico y con quien no se tiene una relación de noviazgo, convivencia o matrimonio) y trabajador/a sexual (persona con la que mantienen relaciones sexuales mutuamente consentidas a cambio de un pago económico). Para acotar el concepto y abreviar el cuestionario, se excluyó besos, caricias, frotamiento y la estimulación manual de los genitales de la pareja, al no implicar riesgo de infectarse del $\mathrm{VIH}$ o ser este riesgo mínimo ${ }^{4}$.

Así, la definición se enfocó a aspectos de riesgo para infectarse de una ITS o del $\mathrm{VIH}$. Al desconocerse las propiedades psicométricas del cuestionario, este estudio tiene como objetivos: 1) describir la distribución del número de parejas sexuales, 2) estudiar las distribuciones, propiedades de discriminación y consistencia interna de los ítems de las dos escalas, 3) determinar la estructura factorial de las dos escalas, y 4) estimar la consistencia interna y describir las distribuciones de las escalas y sus factores.

\section{MÉTODO}

Se realizó una investigación instrumental al centrarse el estudio en el análisis de las propiedades psicométricas de un instrumento de medida. Su diseño fue ex post facto y de corte transversal, al no manipularse variables y colectarse datos de cada participante en un único momento ${ }^{12}$.

Se empleó el muestreo dirigido por entrevistados, el cual se refiere a un tipo de muestreo no probabilístico diseñado para poblaciones ocultas. Los criterios de inclusión fueron: ser hombre, dar el consentimiento informado, ser mayor de edad, ser sexualmente activo y haber tenido al menos una vez sexo con otro hombre. El criterio de eliminación fue: informar ser $\mathrm{VIH}$ positivo.

La muestra quedó integrada por $133 \mathrm{HSH}$ residentes en Monterrey y su área metropolitana, México. La edad varió de 18 a 49 años con una media de 27.11 (DE = 7). Los Journal Health NPEPS. 2018 jul-dez; 3(2):327-351. 
Valdez-Montero C, Rubia JM, Onofre-Rodríguez DJ, Benavides-Torres RA, Ahumada-Cortez JG. Validación...

años de escolaridad variaron de 3 a 25 años con una media de 15.32 (DE = 3.60). Con respecto al trabajo, 95 de los encuestados (71.4\%) dijeron ser laboralmente activos y 38 (28.6\%) estar estudiando. Los ingresos mensuales variaron de 0 a 75,000 pesos con una media de 6,135 ( $D E=7,815)$. Con respecto a tener una pareja estable, 63 de los 133 hombres (47.4\%) indicaron una de sexo masculino, 9 (6.8\%) una de sexo femenino y 61 (45.8\%) ninguna. De los 133 participantes, 87 (65.4\%) se definieron como homosexuales, 19 (14.3\%) bisexuales, 16 (12\%) heterosexuales, 7 (5.3\%) travestis y 4 (3\%) transexuales.

El Cuestionario de Conductas Sexuales en HSH (CCS_HSH) fue elaborado por los autores (véase Figura 1). Se partió de la ESSM en el Matrimonio ${ }^{13}$. Primero se seleccionaron y adaptaron 23 items de la ESSM al contexto de HSH. A continuación, estos 23 ítems se sometieron al juicio crítico de cinco expertos para obtener la redacción final.

Los cinco expertos contaban con grado de doctorado en áreas de conocimiento de ciencias de la salud (psicología o enfermería) y realizaron un juicio crítico de manera individual para evaluar dos aspectos: concordancia (el ítem mide el constructo) y relevancia (su inclusión es necesaria para su medición). Los jueces evaluaron los ítems con una puntuación de 1 a 4 , donde 1 se refería a falta de concordancia o relevancia y 4 mostraba completa concordancia o total relevancia. Se calculó una media para cada uno de los ítems. Se requería una media de al menos 3 en ambas características para que un ítem fuese incluido.

El cuestionario quedó integrado por dos escalas y una pregunta sobre el número de parejas sexuales con cinco categorías ordinales (1-2, 4-5, 6-7, 8-9 y10 o más parejas).

Una escala con ocho ítems con un formato tipo Likert evalúa la frecuencia con que se tienen cuatro tipos de conductas sexuales con mujeres: coito vaginal, sexo oral activo, sexo oral pasivo y coito anal insertivo; así como la frecuencia con que se tienen cuatro tipos de conductas sexuales con hombres: sexo oral activo, sexo oral pasivo, coito anal insertivo y coito anal receptivo. La frecuencia de conductas sexuales se reporta en un rango de cinco categorías ordenadas, las cuales se puntuaron del siguiente modo: 1 = "no he tenido", 2 = "rara vez", 3 = "algunas veces", 4 = "frecuentemente" y 5 = "siempre".

La otra escala con 15 ítems con un formato tipo Likert evalúa la frecuencia con que se usa el condón al tener cinco tipos de conductas sexuales (coito vaginal, sexo oral activo, sexo oral pasivo, coito anal insertivo y coito anal receptivo) con tres tipos de pareja, ya Journal Health NPEPS. 2018 jul-dez; 3(2):327-351. 
Valdez-Montero C, Rubia JM, Onofre-Rodríguez DJ, Benavides-Torres RA, Ahumada-Cortez JG. Validación...

sea masculina o femenina (pareja estable, parejas ocasionales y trabajadores/as sexuales). Primero se indica si ha tenido o no ese tipo de sexo". En el caso de que la respuesta sea si, se señalaba la frecuencia del uso del condón en un rango de cinco categorías ordenadas, las cuales se puntuaron del siguiente modo: 5 = "nunca", $4=$ "a veces", $3=$ "con frecuencia", 2 = "con mucha frecuencia" y 1 = "siempre". En el caso de que sea no, lo que varió de 14 a 16 casos, se dio un valor de 0 . De este modo, se evalúa el grado de desprotección. A mayor puntuación, indica más desprotección.

El estudio fue autorizado por las comisiones de Investigación y Ética en Investigación de la Facultad de Enfermería de la Universidad Autónoma de Nuevo León. El cuestionario fue diseñado y estaba disponible en la plataforma SurveyMonkey. Iniciaba con la hoja de consentimiento informado y las preguntas-filtro (criterios de inclusión). De acuerdo a las normas éticas de investigación de la American Psychological Association ${ }^{14}$, se garantizó el anonimato y la confidencialidad de la información dada; y se proporcionó un correo electrónico en caso de que el participante tuviera preguntas y éstas fueron respondidas por la investigadora principal.

Las variables analizadas en este estudio se pueden clasificar en dos tipos por su escala de medida ${ }^{15}$. Por una parte, se tienen los ítems del cuestionario que son variables en una escala de medida ordinal (con cinco categorías ordenadas), por otra parte, se tienen las puntuaciones en las escalas y sus factores (obtenidas como cociente entre la suma de los ítems y el número de ítems sumados) que son variables cuantitativas continuas.

Con respecto al primer objetivo de describir la distribución del número de parejas sexuales (ítem 3), se calculó la mediana (Mdn) como estadístico de tendencia central y el rango semi-intercuartílico (RSI) como estadístico de variación, al ser una variable con una escala de medida ordinal con cinco categorías ordenadas: 1-2, 4-5, 6-7, 8-9 y 10 o más.

1. ¿Alguna vez ha tenido sexo con una mujer? Si__ No Si la respuesta es Sí, por favor conteste a lo siguiente. Si la respuesta es No pase a la pregunta 2.

\begin{tabular}{|r|c|c|c|c|c|}
\hline Con mujeres & $\begin{array}{c}\text { No he } \\
\text { tenido }\end{array}$ & $\begin{array}{c}A \\
\text { veces }\end{array}$ & $\begin{array}{c}\text { Con } \\
\text { frecuencia }\end{array}$ & $\begin{array}{c}\text { Con mucha } \\
\text { frecuencia }\end{array}$ & Siempre \\
\hline Sexo vaginal & & & & & \\
\hline Sexo oral (tu boca en su vagina) & & & & & \\
\hline Sexo oral (su boca en tu pene) & & & & & \\
\hline Sexo anal (tu pene en su ano) & & & & \\
\hline
\end{tabular}


Valdez-Montero C, Rubia JM, Onofre-Rodríguez DJ, Benavides-Torres RA, Ahumada-Cortez JG. Validación...

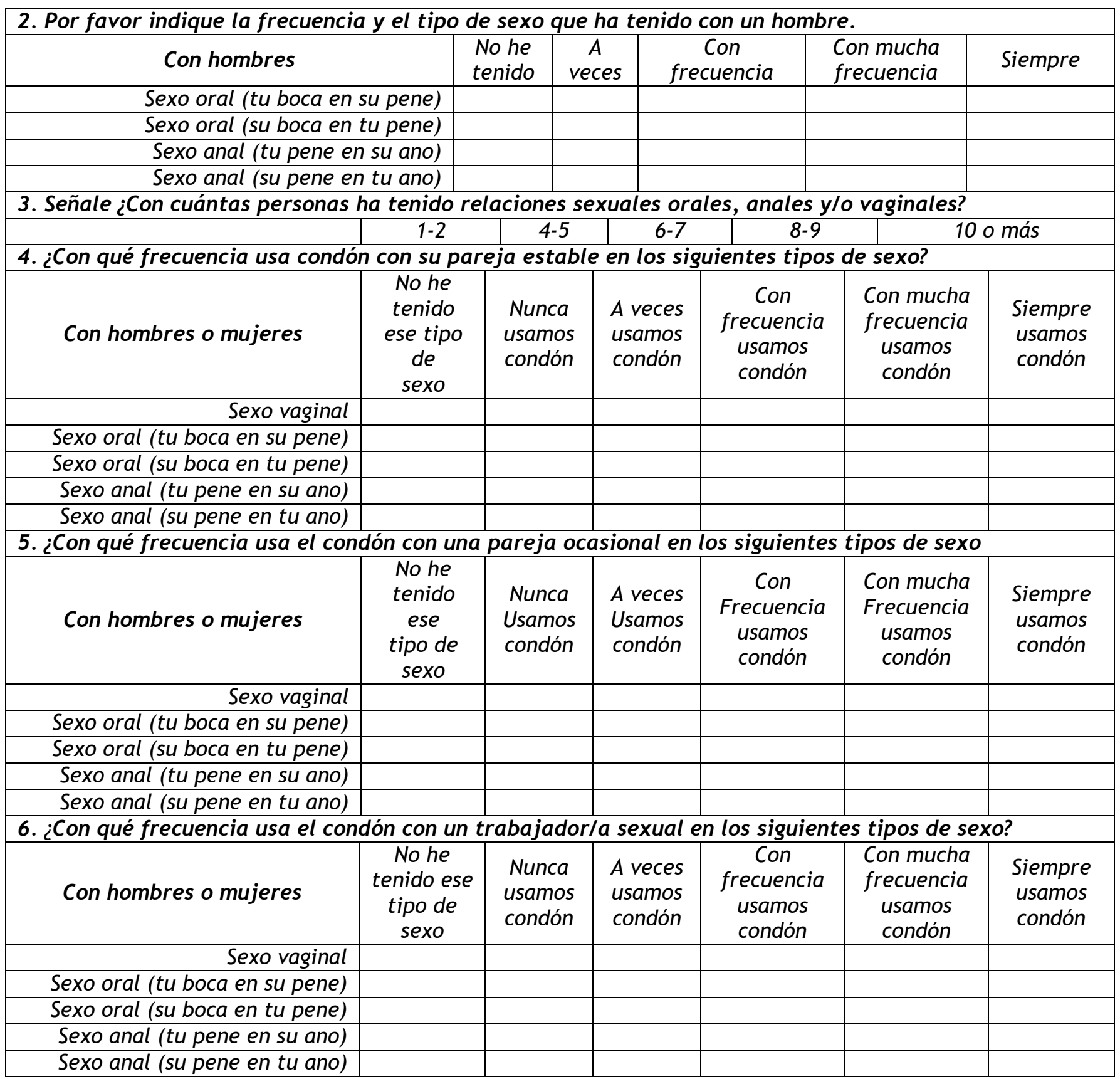

Figura 1 - Cuestionario de conductas sexuales en hombres que tienen sexo con hombres.

Referente al segundo objetivo de estudiar los ítems (variables ordinales), sus distribuciones se describieron por las frecuencias y porcentajes de sus categorías ordenadas, la mediana (tendencia central) y rango semi-intercuartílico (variación). La discriminabilidad de cada ítem se determinó por la diferencia significativa de tendencia central en el ítem entre el grupo de puntuaciones altas y bajas en la escala. Las puntuaciones en la escala se crearon por la suma simple de los ítems. Los grupos se Journal Health NPEPS. 2018 jul-dez; 3(2):327-351. 
Valdez-Montero C, Rubia JM, Onofre-Rodríguez DJ, Benavides-Torres RA, Ahumada-Cortez JG. Validación...

definieron por el primer cuartil y el tercero en la escala. La diferencia de tendencia central se contrastó por la prueba $U$ de Mann-Whitney.

La consistencia interna se estimó por la correlación del ítem (i) con el resto de la escala o suma simple de los ítems excluido el ítem ( $t$-i) y por el efecto de la eliminación del ítem sobre el valor de la consistencia interna de la escala. La correlación se calculó por el coeficiente de correlación poliserial ( $\left.r_{P C}[i, t-i]\right)$, al correlacionarse variables ordinales (i) con variables cuantitativas ( $t-i)$. La consistencia interna se estimó por el coeficiente alfa ordinal ( $a_{t-i}$ ordinal). Si $r_{P C}[i, t-j]<.30 \mathrm{y} / 0$ se incrementa la consistencia interna de la escala con la eliminación del ítem ( $a_{t-i}$ ordinal $>a_{t}$ ordinal), se consideró que el ítem presenta problemas de consistencia interna ${ }^{16}$.

Con respecto al tercer objetivo de determinar la estructura factorial, se usó análisis factorial exploratorio (AFE) y confirmatorio (AFC). Ambos análisis se ejecutaron en la misma muestra de 133 participantes sobre la matriz de correlaciones policóricas (rPC). Estas correlaciones fueron estimadas por el método de Dos Pasos para Máxima Verosimilitud. En el AFE, el número de factores se determinó por los criterios de Horn (percentil 95 como criterio de intersección, datos generados por permutaciones bajo un modelo de componentes principales, con 500 muestras simuladas) y Velicer (medias de las correlaciones parciales al cuadrado). Los factores se extrajeron por el método de Mínimos Cuadrados no Ponderados (ULS). La matriz factorial se rotó por el método Promax. En el $A F C$, la función de discrepancia se minimizó por ULS. Se contemplaron seis índices de ajuste: chi-cuadrada relativa $\left(\mathrm{x}^{2} / \mathrm{gl}\right)$, índice de bondad de ajuste (GFI) y su modalidad corregida (AGFI) de Jöreskog y Sörbom, índice normado de ajuste (NFI) de Bentler y Bonett, índice relativo de ajuste (RFI) de Bollen y el residuo estandarizado cuadrático medio (SRMR). Se estipularon como valores de buen ajuste para los índices: $x^{2} / g l \leq 2, G F I$, $N F I$ y $R F I \geq .95, A G F I \geq .90$ y SRMR $\leq .05 ;$ y como valores adecuados: $x^{2} / g l \leq 3$, GFI, NFI y RFI $\geq .90, A G F I \geq .85$ y $S R M R<.10^{17}$. Al aplicarse el AFC sobre la misma muestra en la que se hizo el AFE, se trata de un análisis complementario al exploratorio para reportar la bondad de ajuste del modelo en la muestra, pero no es propiamente un contraste o evidencia adicional de validez de constructo ${ }^{16}$.

Con respecto al cuarto objetivo, los valores de consistencia interna entre los ítems de las escalas y sus factores (variables ordinales) se calcularon por el coeficiente alfa Journal Health NPEPS. 2018 jul-dez; 3(2):327-351. 
Valdez-Montero C, Rubia JM, Onofre-Rodríguez DJ, Benavides-Torres RA, Ahumada-Cortez JG. Validación...

ordinal (a ordinal). Se interpretó que valores de a ordinal $<.50$ evidencian una consistencia interna inaceptable, de .50 a .59 pobre, de .60 a .69 cuestionable, de .70 a .79 aceptable, de .80 a .89 buena $y \geq .90$ excelente $^{18}$. Se describió las distribuciones de las puntuaciones en escalas y sus factores (variables cuantitativas) por medias, desviaciones estándar y cuantiles, y se contrastó su ajuste a la normalidad por la prueba de JarqueBera. Los cálculos se realizaron con SPSS21, menú $R 2.4$ para SPSS21 y AMOS16.

\section{RESULTADOS}

De los 129 participantes que respondieron a la pregunta, 52 (39\%) dijeron haber tenido 10 o más parejas sexuales, 25 (19\%) 4 o 5, 20 (18\%) 1 o 2, 20 (15\%) 8 o 9 y 12 (9\%) 6 o 7. La mediana correspondió al cuarto valor ("8 o 9") y el recorrido semiintercuartílico fue 1.5 .

Distribución, discriminabilidad y consistencia interna de los ocho ítems de frecuencia de relaciones sexuales

Las medianas de sexo vaginal, oral y anal con mujeres coincidieron en uno ("no he tenido"). De los 131 hombres que respondieron a la pregunta sobre relaciones sexuales con mujeres, 76 (58\%) no han tenido coito vaginal, 87 (66\%) sexo oral pasivo, 97 (74\%) sexo oral activo y 107 (82\%) coito anal. Las medianas de sexo oral activo y pasivo con hombres coincidieron en tres ("con frecuencia") y las de coito anal insertivo y receptivo coincidieron en dos ("a veces").

Sólo el ítem de sexo oral activo con mujeres no fue discriminativo, además tuvo

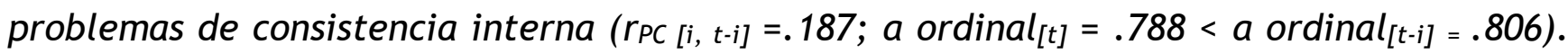
El ítem de coito vaginal tuvo una correlación poliserial con el resto de la escala baja (rPC [i, $\left.t_{-i]}=.187\right)$, aunque su eliminación hizo disminuir la consistencia interna de la escala. La eliminación del ítem de coito anal receptivo con hombres incrementó la consistencia interna de la escala (de a ordinal $[t]=.788$ a a ordinal $[t-i]=.797$ ), aunque su correlación con el resto de la escala fue moderada $\left(r_{P C}[i, t-i]=.480\right)$. 
Valdez-Montero C, Rubia JM, Onofre-Rodríguez DJ, Benavides-Torres RA, Ahumada-Cortez JG. Validación...

El número de factores convergió en dos por el criterio de Horn y Velicer para los ocho ítems de frecuencia de relaciones sexuales. Al extraerse dos factores, se explicó el $85 \%$ de la varianza total. Tras la rotación se configuró un primer factor con cuatro ítems sobre relaciones sexuales con hombres con consistencia interna buena (a ordinal $=.898$ ). El segundo factor quedó definido por los cuatro ítems sobre relaciones sexuales con mujeres con consistencia interna excelente (a ordinal $=.927$ ). La correlación entre ambos factores fue muy débil y no significativa $(r=.009, p=.922)$.

Al resultar los dos factores independientes y al haber problemas de discriminación y consistencia interna en el ítem de coito vaginal del factor de sexo con mujeres y debilidades de consistencia interna en un segundo ítem del factor de sexo con mujer (coito anal) y en otro ítem del factor de sexo con hombres (coito anal receptivo), se optó por definir dos escalas.

Los cuatro ítems de frecuencia de relaciones sexuales con mujeres fueron discriminativos y sin problemas de consistencia interna dentro de su escala. El número de factores fue uno por el criterio de Horn y dos por el criterio de Velicer para estos cuatro items. Al extraer un factor, las cargas factoriales variaron de .790 a .941 y se explicó el $76 \%$ de la varianza total. La consistencia interna de los cuatro ítems de sexo con mujeres fue excelente (a ordinal $=$.926).

Al extraer dos factores siguiendo el criterio de Velicer, se explicó el 95\% de la varianza total. Tras la rotación se definió un primer factor con los ítems de coito vaginal y sexo oral activo o de rol caballeroso con la mujer con consistencia interna excelente (a ordinal $=.973$ ), y un segundo factor con los ítems de sexo oral pasivo y coito anal o de rol dominante con la mujer con consistencia interna excelente (a ordinal = .946). La correlación de ambos factores fue alta y negativa $(r=-.685, p<.001)$.

Los cuatro items de frecuencia de relaciones sexuales con hombres fueron discriminativos y sin problemas de consistencia interna dentro de su escala. El número de factores fue uno por el criterio de Horn y dos por el criterio de Velicer para estos cuatro ítems. Al extraer un factor, las cargas factoriales variaron de .733 a .912 y se explicó el $70 \%$ de la varianza total. La consistencia interna de los cuatro ítems de sexo con los hombres fue buena (a ordinal $=.898$ ). 
Valdez-Montero C, Rubia JM, Onofre-Rodríguez DJ, Benavides-Torres RA, Ahumada-Cortez JG. Validación...

Al extraer dos factores siguiendo el criterio de Velicer, se explicó el $87 \%$ de la varianza total. Tras la rotación se definió un primer factor con los ítems de sexo oral pasivo y coito anal insertivo o rol homosexual activo con consistencia interna excelente ( $a$ ordinal $=.937$ ) y un segundo factor con los ítems de sexo oral activo y coito anal receptivo o rol homosexual pasivo con consistencia interna excelente (a ordinal $=.907)$. La correlación de ambos factores fue alta y positiva $(r=.655, p<.001)$.

Se especificó un modelo de un factor de sexo con mujeres con cuatro indicadores. Los pesos factoriales variaron de .790 a .941 con una media de .871 (véase Figura 2). Tres de los seis índices de ajuste fueron buenos $(G F I=.985, A G F I=.923$ y NFI $=.976)$, dos aceptables $(R F I=.928$ y $S R M R=.092)$ y uno malo $\left(x^{2} / g l=5.552\right)$.

Retomando el modelo bifactorial del análisis factorial exploratorio, se consideró el rol dominante con la mujer, así se introdujo una correlación entre los residuos de medida de sexo oral pasivo y coito anal. Tras esta modificación, el ajuste fue bueno por los seis índices contemplados $\left(\mathrm{x}^{2} / \mathrm{gl}=0.228, \mathrm{GFI}=1, \mathrm{AGFI}=.997, \mathrm{NFI}=.999, \mathrm{RFI}=.997\right.$ y $S R M R=$ .014). Si se asumiera normalidad multivariada, la bondad de ajuste se mantendría por la prueba chi-cuadrado $\left(x^{2}[1, N=131], p=.594\right)$ y sería significativamente mejor que la del modelo con los residuos independientes $\left(\Delta x^{2}[1, N=131]=10.865, p<.001\right)$.

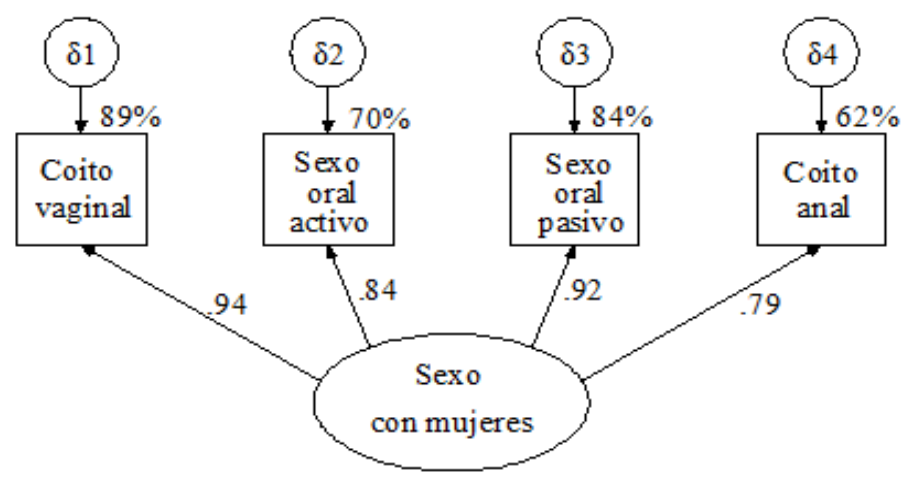

Figura 2. Modelo estandarizado de un factor de sexo con mujeres.

Se especificó un modelo de un factor de sexo con hombres con cuatro indicadores. Los pesos factoriales variaron de .733 a .912 con una media de .831 (véase Figura 3). Tres de los seis índices de ajuste fueron buenos $(G F I=.982, A G F I=.912$ y NFI $=.970)$, dos aceptables $(R F I=.911$ y $S R M R=.094)$ y uno malo $\left(x^{2} / g l=5.255\right)$. Retomando el modelo bifactorial del análisis factorial exploratorio, se consideró el rol homosexual activo, así se 
Valdez-Montero C, Rubia JM, Onofre-Rodríguez DJ, Benavides-Torres RA, Ahumada-Cortez JG. Validación...

introdujo una correlación entre los residuos de medida de sexo oral pasivo y coito anal insertivo.

Tras esta modificación, el ajuste fue bueno por los seis índices contemplados $\left(x^{2} / g l\right.$ $=0.008, \mathrm{GFI}=1, \mathrm{AGFI}=1, \mathrm{NFI}=1, R F I=1$ y $S R M R=.003)$. Si se asumiera normalidad multivariada, la bondad de ajuste se mantendría por la prueba chi-cuadrado ( $x^{2}[1, N=121]$ $=0.008, p=.929)$ y sería significativamente mejor que la del modelo con los residuos independientes $\left(\Delta x^{2}[1, N=121]=10.501, p=.001\right)$.

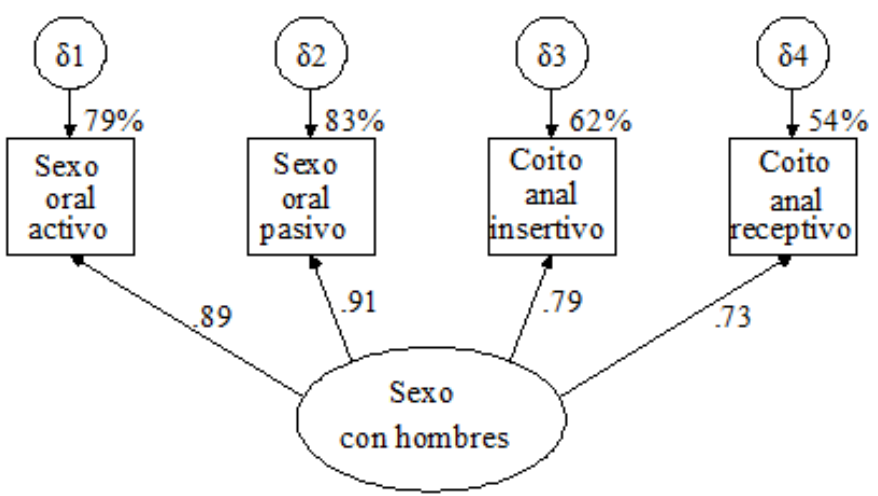

Figura 3 - Modelo estandarizado de un factor de sexo con hombres.

Distribución de las puntuaciones en las escalas y factores de frecuencia de relaciones sexuales con mujeres o con hombres

Para obtener las puntuaciones de las dos escalas de frecuencia de relaciones sexuales (con mujeres o con hombres), se dividió la suma simple de ítems por el número de items sumados en cada escala. La distribución de la escala de conductas sexuales con hombres $(M=2.940$ [IC 95\%: $2.717,3.163]$ y $D E=1.237)$ se ajustó a la normalidad $(J B=$ 5.816, $p>.05)$. La distribución de la escala de conductas sexuales con mujeres ( $M=1.510$ [IC 95\%: $1.374,1.646]$ y $D E=.787)$ fue simétrica positiva $(S=1.873, E E=.212)$, pero leptocúrtica $(C=3.537, E E=.420)$, no ajustándose a la normalidad $(J B=144.880, p<.001)$; su mediana y primer cuartil correspondieron a 1, el tercer cuartil a 1.75 y el percentil 80 a 2.

Distribución, discriminabilidad y consistencia interna de los 15 ítems de sexo desprotegido 
Valdez-Montero C, Rubia JM, Onofre-Rodríguez DJ, Benavides-Torres RA, Ahumada-Cortez JG. Validación...

Las medianas de los ítems de sexo desprotegido al practicar coito vaginal con los tres tipos de parejas, así como las de sexo oral activo y pasivo y coito anal insertivo y receptivo con trabajador/ a sexual coincidieron en cero ("no he tenido ese tipo de sexo"). Las medianas de sexo con parejas ocasionales, salvo coito vaginal, así como coito anal insertivo y receptivo con pareja estable coincidieron en dos ("con mucha frecuencia"). Las medianas de sexo oral activo y pasivo con pareja estable coincidieron en cuatro ("a veces").

Los ítems de sexo desprotegido al practicar coito vaginal con pareja estable ( $Z_{U}=$ $0.650, p>.05)$ y con parejas ocasionales $\left(Z_{U}=-1.894, p>.05\right)$ no tuvieron capacidad discriminativa. También estos dos ítems mostraron problemas de consistencia interna con correlaciones con el resto de la escala menores que .30 e incremento de la consistencia interna con su eliminación, cuyo valor fue .863 para los 15 ítems ( $r_{P C}[i, t-i]=.083$ y a ordinal $_{[t-i]}=.869$ en coito vaginal con pareja estable, y $r_{P C}[i, t-i]=.128$ y a ordinal $[t-i]=.863$ en coito vaginal con parejas ocasionales). Debido a los problemas de discriminación y consistencia interna se eliminaron.

\section{Estructura factorial de los 13 ítems seleccionados de sexo desprotegido}

El número de factores fue tres por el criterio de Horn y cuatro por el criterio de Velicer para los 13 ítems. Al extraer tres factores, se explicó el 79\% de la varianza total. Tras la rotación, el primer factor quedó configurado por los cinco tipos de relaciones sexuales desprotegidas con trabajadores/ras sexuales con cargas altas. La consistencia interna de los cinco ítems fue excelente (a ordinal $=.961$ ).

El segundo factor quedó configurado por cuatro ítems de sexo desprotegido al practicar sexo oral y coito anal con parejas ocasionales. La consistencia interna de los cuatro ítems fue buena (a ordinal $=.849$ ). El tercer factor quedó configurado por cuatro ítems de sexo desprotegido al practicar sexo oral y coito anal con pareja estable. La consistencia interna de los cuatro ítems fue buena ( $a$ ordinal $=.858$ ).

La correlación del primer factor fue moderada con el segundo $(r=.304, p=.001)$ y muy débil y no significativa con el tercero $(r=.021, p=.825)$. La correlación entre el 
Valdez-Montero C, Rubia JM, Onofre-Rodríguez DJ, Benavides-Torres RA, Ahumada-Cortez JG. Validación...

segundo y el tercero fue moderada $(r=.370, p<.001)$. Al extraer cuatro factores, el cuarto factor quedó definido por un solo ítem, por lo que se desestimó esta solución.

Se especificó un modelo de tres factores correlacionados: sexo desprotegido con trabajadores/as sexuales con cinco indicadores, con parejas ocasionales con cuatro indicadores y con pareja estable con cuatro indicadores. Los pesos estandarizados de medida variaron de .516 a .985 con un promedio de .823 .

La correlación del factor de sexo desprotegido con trabajadores/as sexuales fue moderada con el factor de sexo desprotegido con parejas ocasionales $(r=.432)$, pero muy débil con el factor de sexo desprotegido con pareja estable $(r=.097)$. La correlación entre los factores de sexo desprotegido con parejas ocasionales y con pareja estable fue alta ( $r=$ .525) (véase Figura 4).

La bondad de ajuste fue buena por cinco índices $\left(x^{2} / g l=1.964, G F I=.952, A G F I=\right.$ .929 y $R F I=.915)$ y adecuada por dos $(N F I=.939$ y $S R M R=.091)$. Si se asumiera normalidad multivariada, la bondad de ajuste mantendría por el estadístico chi-cuadrado $\left(x^{2}[62, N=113]=\right.$ 121.770, $p=.476)$.

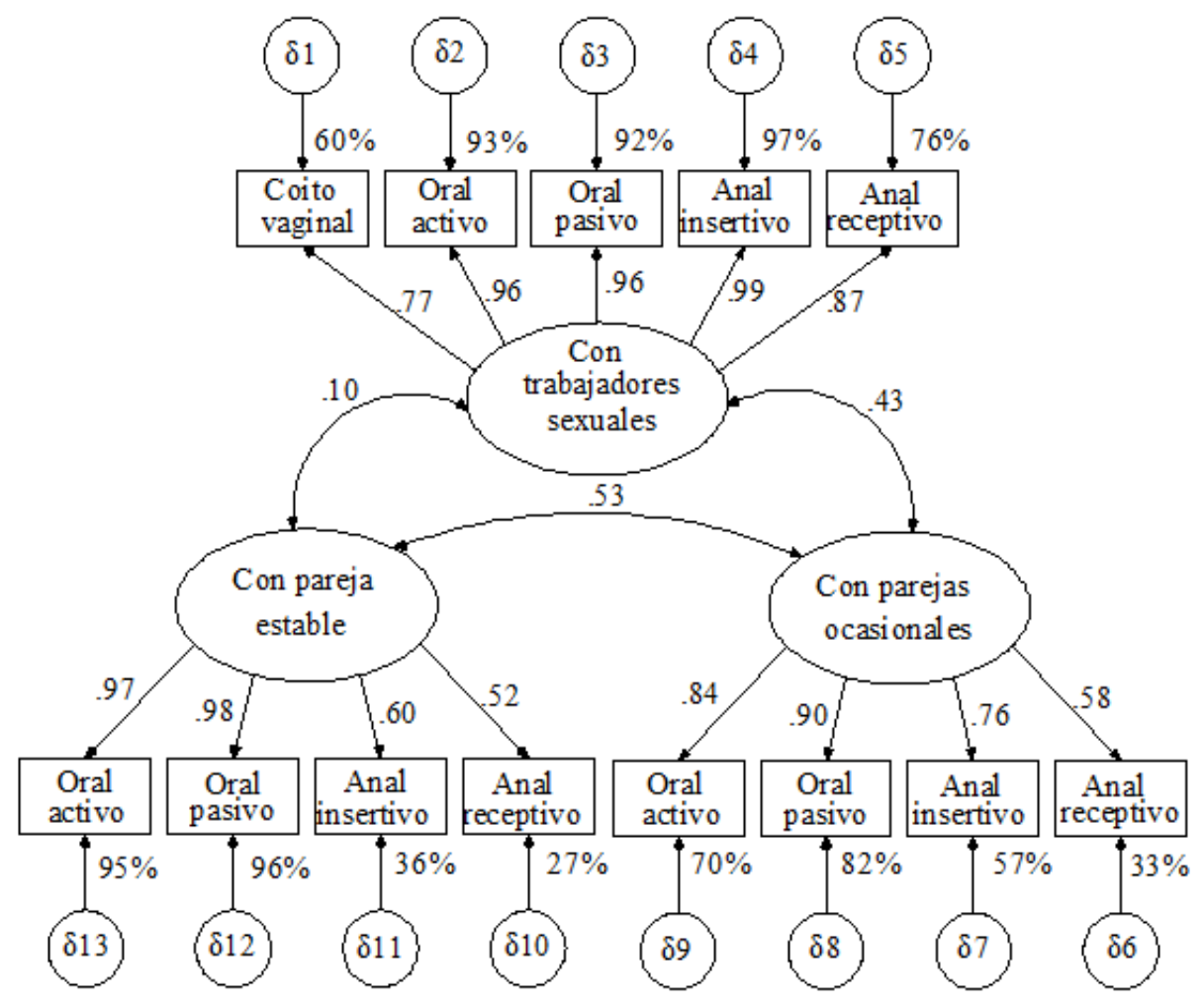

Figura 4 - Modelo estandarizado de tres factores correlacionados de sexo desprotegido. 
Valdez-Montero C, Rubia JM, Onofre-Rodríguez DJ, Benavides-Torres RA, Ahumada-Cortez JG. Validación...

\section{Distribución de las puntuaciones en las escalas y factores de sexo desprotegido}

Para crear las puntuaciones en la escala de sexo desprotegido y sus tres factores se asignó cero a la respuesta "no he tenido ese tipo de sexo", uno a la respuesta "siempre usamos condón", dos a la respuesta "con mucha frecuencia", tres a la respuesta "con frecuencia", cuatro a la respuesta "a veces" y cinco a la respuesta "nunca". Al ítem de coito vaginal se les ponderó por 1.5, a los tres ítems de coito anal insertivo por 2 y a los tres ítems de sexo anal receptivo por 2.5. Para conservar esta ponderación se crearon puntuaciones en forma de índices. La puntuación total y las de los factores se obtienen multiplicando por 100 el cociente entre la suma ponderada de los ítems y la puntuación máxima posible (105 para la puntuación total, 32.5 para sexo desprotegido con parejas ocasionales y trabajadores/as sexuales y 40 para sexo desprotegido con pareja estable). El rango de las puntuaciones en escala y sus tres factores varía en un continuo de 0 a 100. Una mayor puntuación, refleja más mayor frecuencia de sexo desprotegido.

La distribución del índice total de sexo desprotegido ( $M=35.959$ [IC 95\%: 33.027, 38.890] y $D E=15.728)$ se ajustó a un modelo de curva normal $(J B=2.376, p>.05)$, la del índice de sexo desprotegido con pareja estable $(M=53.683$ [IC 95\%: 48.979, 58.387] y $D E=$ 25.236), también ajustó a la normalidad $(J B=0.390, p>.05)$, al igual que la del índice de sexo desprotegido con parejas ocasionales $(M=42.914$ [IC 95\%: 38.808, 47.019] y $D E=$ 22.028; $J B=1.135, p>.05)$. La distribución del índice de sexo desprotegido con trabajadores/as sexuales $(M=15.907$ [IC 95\%: $11.312,20.503$ ] y $D E=24.655)$ fue simétrica positiva $(S=1.113, E E=.227)$ y mesocúrtica $(C=-0.353, E E=.451)$, y no se ajustó $a$ un modelo de curva normal $(J B=23.917, p<.01)$; su mediana y primer cuartil correspondieron a 0 , el tercer cuartil a 39.375 y el percentil 80 a 41.5.

\section{DISCUSIÓN}

El número de parejas sexuales entre los participantes fue alto conforme con los estudios hechos en población mexicana ${ }^{19}$ y en otros países, ya sean desarrollados, como Rusia, Estados Unidos y Canadá, en vías de desarrollo, como los países centroamericanas y sudamericanos, o subdesarrollados, como los países subsaharianos, ${ }^{20}$ lo que constituye uno de los motivos por lo que representan el principal grupo de riesgo para infectarse de ITS y del $\mathrm{VIH}^{21}$. La mediana fue de ocho a nueve parejas sexuales en una distribución con sesgo Journal Health NPEPS. 2018 jul-dez; 3(2):327-351. 
Valdez-Montero C, Rubia JM, Onofre-Rodríguez DJ, Benavides-Torres RA, Ahumada-Cortez JG. Validación...

hacia la categoría ordinal más alta ("10 o más"). Debe señalarse que es frecuente que en las encuestas sobre sexualidad aplicadas a esta población, el número de parejas sexuales se ha dicotomizado usando como criterio 10 parejas ${ }^{22}$. Este punto de corte es concordante con nuestros datos, el tenerse 10 o más parejas sexuales se puede interpretar como un número alto de parejas sexuales.

Si la escala de frecuencia de relaciones sexuales con mujeres y con hombres es tratada como un conjunto de ocho ítems, se revela que el ítem de sexo oral activo con mujeres no es discriminativo ni consistente; y que los ítems de coito vaginal con mujeres y de coito anal receptivo con hombres tienen debilidades de consistencia interna. No obstante, si se divide en dos escalas independientes de cuatro ítems (sexo con hombres y sexo con mujeres), desaparecen estos problemas. Esta separación no sólo se justifica por el análisis de las propiedades de los ítems, sino que se fundamenta en el análisis factorial. El número de factores con los ocho ítems fue de dos por los criterios de Horn y Velicer, y estos dos factores fueron independientes. Al contrastarse modelos unidimensionales con cuatro indicadores, se logró un ajuste de los datos aceptable; el modelo de dos factores con cuatro indicadores, cada uno tuvo mal ajuste a los datos. Esta unidimensionalidad se sustentó en el criterio de Horn que es más exacto que el de Velicer ${ }^{16,23 .}$

De aquí se deriva que una mayor frecuencia de relaciones sexuales con un género no implica tener menos frecuencia de relaciones sexuales con el otro género entre los participantes que en su mayoría se definieron como homosexuales, sino que ambas frecuencias son independientes.

El análisis factorial exploratorio de los dos conjuntos de cuatro ítems, guiado por el criterio de Velicer, reveló un patrón de covarianza: la diferenciación de un rol de dominancia/sumisión con hombres o rol homosexual activo/pasivo, y de dominancia/caballerosidad con mujeres. La correlación entre los roles homosexuales activo y pasivo fue alta y positiva, indicando que ambos roles se intercambian al tener relaciones sexuales con hombres. Sin embargo, la correlación entre los factores de rol caballeroso con la mujer y rol dominante fue alta y negativa, lo que indica que se adopta un rol en contraposición al otro, pero no se intercambian al tener relaciones sexuales con mujeres. Estas correlaciones de distinto signo podría indicar una mayor libertad con respecto a los 
Valdez-Montero C, Rubia JM, Onofre-Rodríguez DJ, Benavides-Torres RA, Ahumada-Cortez JG. Validación...

roles tradicionales de género al tenerse relaciones sexuales con otros hombres, pero su manifestación en las relaciones heterosexuales.

Al introducir la variante activa en los modelos unidimensionales a través de la correlación entre residuos, se logró un buen ajuste a los datos. Esto indica que el patrón dominante (sexo oral pasivo y coito anal insertivo) es atribuible $a$ un subgrupo de participantes. El patrón se define de forma más rígida frente a las mujeres y de forma más flexible frente a los hombres, sin implicar necesariamente bisexualidad. Cabe interpretar que este patrón en hombres que se identifican como heterosexuales refleja una homosexualidad latente y esta identificación "como hombres" probablemente sea motivado por homonegatividad internalizada ${ }^{24}$.

Concordante con la identidad homosexual en dos tercios de los participantes, la frecuencia de relaciones sexuales fue significativamente más alta con hombres que con mujeres. Al tener sexo con hombres, se practica más sexo oral activo y pasivo, y menos coito anal insertivo y receptivo. Al tener sexo con mujeres, se practica más coito vaginal y cunnilingus, y menos la felación y el coito anal insertivo. Por consiguiente, la actividad sexual más frecuente entre los entrevistados fue el sexo oral con hombres, seguido del sexo anal con hombres y la menos frecuente fue el sexo anal con mujeres. Estos datos concuerdan con estudios sobre conducta sexual en $\mathrm{HSH}$ realizados en otros países como Brasil ${ }^{25}$ y Australia ${ }^{26}$.

Aunque el ítem de sexo anal con mujeres mostró un sesgo muy marcado hacia el valor mínimo, no llega a ser un caso de efecto suelo (valor mínimo $\geq 90 \%$ de la distribución) y resultó discriminativo y consistente, por lo que debe conservarse en la escala de frecuencia de relaciones sexuales con mujeres.

Ambas escalas evalúan frecuencia de conductas sexuales, ya que el riesgo está mediado por sexo desprotegido (sin uso del condón) y al tenerse de la forma específica dentro del cuestionario una escala para evaluar sexo desprotegido, se decidió no ponderar las conductas. Dividido el rango de posibles valores continuos $([1,5])$ en cinco intervalos de amplitud constante (0.8), la media de la escala de conductas sexuales con hombres correspondió al tercer intervalo $([2.601,3.4] \rightarrow 3=$ "con frecuencia") y la media de la escala de conductas sexuales con mujeres correspondió al primer intervalo $([1,1.8] \rightarrow 1=$ 
Valdez-Montero C, Rubia JM, Onofre-Rodríguez DJ, Benavides-Torres RA, Ahumada-Cortez JG. Validación...

"nunca"), lo que revela que el participante promedio es sexualmente muy activo con hombres, pero no con mujeres.

Además, la distribución normal de la escala de conducta sexual con hombres, refleja que el rasgo evaluado es característico y con una expresión variable de individuo a individuo dentro de la población de la que fue extraída la muestra; cuando la distribución tiene una asimetría negativa de la escala de conducta sexual con mujeres, indica que es un rasgo no característico.

Las puntuaciones en la escala de sexo con hombres pueden baremarse por medio de la media y la desviación estándar, al ajustarse su distribución a una curva normal; y las puntuaciones en la escala de sexo con mujeres por medio de puntuaciones de percentil, al desviarse su distribución de un modelo de curva gaussiana ${ }^{27}$. Entre los participantes, puntuaciones $\geq 4.177(M+D E)$ reflejan actividad sexual excesiva con hombres y $\geq 2$ (percentil 80) con mujeres.

Con respecto a la escala de sexo desprotegido, los dos ítems referentes al sexo vaginal con parejas ocasionales y con pareja estable deben ser eliminados por problemas de consistencia interna y para definir una estructura de tres factores con consistencia interna alta o excelente y ajuste a los datos de aceptable a bueno: sexo desprotegido con pareja estable, con parejas ocasionales y con trabajadores sexuales. Las correlaciones entre los tres factores y medias de los ítems indican que las personas con pareja estable muy rara vez pagan por tener sexo, y aquellos sin pareja estable son los que acuden a estos servicios. Conforme con los datos de Arreola y colaboradores ${ }^{28}$, estos servicios son con travestis, transexuales y hombres, más que con mujeres.

Las distribuciones de la escala de sexo desprotegido y las de sus factores (con pareja estable y con parejas ocasionales) se ajustaron a una curva normal, reflejando conductas propias dentro de la población, cuya expresión cuenta con libertad y su regulación está sometida a un criterio personal y un juego de adaptación ambiental. Sin embargo, la distribución del factor de sexo desprotegido con trabajadores/as sexuales mostró sesgo hacia las puntuaciones de sexo seguro y uso del condón con mucha frecuencia, lo que refleja que es una conducta poco característica de la población y regulada por normas más rígidas o impuestas por algún poder externo, que en este caso sería la política socio-sanitaria de control del $\mathrm{VIH}$. 
Valdez-Montero C, Rubia JM, Onofre-Rodríguez DJ, Benavides-Torres RA, Ahumada-Cortez JG. Validación...

La media de la puntuación total de sexo desprotegido revela predomino del sexo protegido o riesgo bajo (> 21 que corresponde al valor máximo al responder a los 13 ítems con la opción "siempre" y $\leq 41.5$ que corresponde al valor máximo al responder a los 13 items con la opción "con mucha frecuencia"). No obstante, debe considerarse que la media del factor de sexo desprotegido con trabajadores/as sexuales se ubicó en el intervalo de dominio del sexo seguro y protegido $(\leq 21)$, pero las medias de los factores de sexo desprotegido con pareja estable y con parejas ocasionales se ubicaron en el intervalo de desprotección o riesgo medio (> 41.5 y $\leq 63$ que es el valor máximo al responder a los 13 items con la opción "con frecuencia"), más próximo al límite inferior con parejas ocasionales (41.501) y al superior con pareja estable (63).

Por lo tanto, el riesgo bajo de la puntuación total da una falsa impresión cuando el riesgo es medio con la pareja estable y con parejas ocasionales. Si se considera que el número de parejas sexuales es alto entre los participantes (dos de cada cinco reportan $10 \mathrm{o}$ más parejas sexuales), que la mayoría de estas parejas son HSH, que entre aquellos con una pareja estable, la pareja concurrente más probable sea una ocasional masculina con la cual se tiene una situación de riesgo medio, siendo la desprotección aún mayor con la pareja estable, entonces se deduce que el riesgo de infectarse de una ITS y de VIH alcanza un nivel que requiere atención desde una política pública de prevención.

Las puntuaciones totales en la escala y las puntuaciones en los dos factores de sexo desprotegido con pareja estable y parejas ocasionales pueden ser baremadas por la media y la desviación estándar al ajustarse sus distribuciones a una curva normal. Entre los participantes las puntuaciones $\geq 51.687$ en la escala, $\geq 78.919$ en el factor de pareja estable $y \geq 64.942$ en el factor de parejas ocasionales, reflejan prácticas de sexo desprotegido con mayor frecuencia que lo usual. Las puntuaciones en el factor de sexo desprotegido con trabajadores sexuales deben baremarse por puntuaciones de percentil al desviarse su distribución de la normalidad ${ }^{27}$. Entre los participantes las puntuaciones $\geq$ 41.5 reflejan una frecuencia significativamente alta de sexo desprotegido.

Como limitación del estudio, se tiene el empleo de un muestreo dirigido por entrevistados por lo oculto de la población, siendo poco viable un muestreo probabilístico. No obstante, las características sociodemográficas de la muestra son muy semejantes al estudio de Gutiérrez ${ }^{29}$ con un muestreo probabilístico de sitios de encuentro en la Ciudad Journal Health NPEPS. 2018 jul-dez; 3(2):327-351. 
Valdez-Montero C, Rubia JM, Onofre-Rodríguez DJ, Benavides-Torres RA, Ahumada-Cortez JG. Validación...

de México. En estos estudios la mayoría de los hombres eran jóvenes, económicamente activos, con niveles de escolaridad y socioeconómico mayores que el promedio de la población general. Una segunda limitación, es el tamaño de la muestra de 133 participantes, por lo que debe considerarse un estudio piloto. Una de las fortalezas del estudio es la selección de pruebas estadísticas apropiadas a los niveles de medida y distribuciones de los ítems y las escalas.

Una tercera limitación del estudio, es en relación con la escala de medida de los ítems. Salvo en la pregunta tres sobre número de parejas, las opciones de respuesta usan criterios de valoración relativamente subjetivos ("no he tenido", "a veces", "con frecuencia", "con mucha frecuencia" y "siempre" para frecuencia de conductas sexuales; "nunca usamos condón”, "a veces usamos condón”, "con frecuencia usamos condón”, "con mucha frecuencia usamos condón" y "siempre usamos condón" para uso de condón). Con criterios de valoración relativamente subjetivos se hace referencia a que son términos de uso común, fáciles de comprender y diferenciables al establecer un orden. Se podrían usar opciones más objetivas. Por ejemplo, se podría plantear para las preguntas 1 y 2: "En las relaciones sexuales que ha tenido con mujeres/hombres, señale en qué porcentaje de veces están presentes las siguientes conductas: 0\%, <25\%, 25\% a 49\%, 50\% a 74\% y $\geq 75 \%$; y

para las preguntas 4, 5 y 6: ¿Cuál es el porcentaje de veces con que usa condón con su pareja estable/una pareja ocasional/sexo servidor(a) en los siguientes tipos de sexo? No he tenido ese tipo de sexo. Sí he tenido: $0 \%,<25 \%, 25 \%$ a $49 \%, 50 \%$ a $74 \%$ y $\geq 75 \%$. No obstante, se consideró que estas opciones son más complejas de responder, especialmente para una persona de baja escolaridad, y están implícitas en las categorías usadas, las cuales son más intuitivas a efectos de cálculo mental ${ }^{30}$.

\section{CONCLUSIÓN}

La distribución del número de parejas sexuales presentó sesgo hacia valores altos, aunque la tendencia central fue menor que 10 parejas. Los ocho ítems de frecuencia de relaciones sexuales con hombres y mujeres definieron dos escalas independientes con consistencia interna buena. Por una parte, se definió el modelo de un factor con cuatro indicadores de sexo con mujeres y por otra parte el modelo de un factor de sexo con hombres. Ambos modelos tuvieron un ajuste a los datos aceptable, al especificar los Journal Health NPEPS. 2018 jul-dez; 3(2):327-351. 
Valdez-Montero C, Rubia JM, Onofre-Rodríguez DJ, Benavides-Torres RA, Ahumada-Cortez JG. Validación...

residuos independientes; y el ajuste fue bueno, una vez que se especificó la correlación entre los residuos un rol dominante con las mujeres (correlación entre los residuos de los ítems de sexo oral pasivo y coito anal) y el rol homosexual activo (correlación entre los residuos de los ítems de sexo oral pasivo y coito anal insertivo).

La distribución de la escala frecuencia de relaciones sexuales con hombres se ajustó a una distribución normal y puede ser baremada por puntuaciones $T$ (media de 50 y desviación estándar de 10) basadas en la media aritmética y desviación estándar. Congruente con el hecho de ser $\mathrm{HSH}$, la distribución de la escala frecuencia de relaciones sexuales con mujeres fue asimétrica positiva, por lo que debe ser baremada a través de puntuaciones de percentil.

Dos de los quince ítems de la escala de sexo desprotegido, los de coito vaginal con parejas ocasionales y con pareja estable, mostraron problemas de consistencia interna y requieren ser eliminados para definirse un modelo de tres factores correlacionados con ajuste a los datos de bueno a aceptable. Un factor es de sexo desprotegido con trabajadores/as sexuales con cinco indicadores con consistencia interna excelente. Otro factor es de sexo desprotegido con parejas ocasionales y otro con pareja estable, ambos con cuatro indicadores con consistencia interna buena.

Las distribuciones de la escala sexo con hombres y de sexo desprotegido y las de los factores de sexo desprotegido con pareja estable y parejas ocasionales se ajustaron a una curva normal y pueden ser baremadas por puntuaciones $T$ basadas en la media aritmética y la desviación estándar. Las distribuciones de la escala de sexo con mujeres y del factor de sexo desprotegido con trabajadores/as sexuales mostraron asimetría positiva y deben ser baremadas por puntuaciones de percentil. Los factores de sexo desprotegido con la pareja estable y parejas ocasionales revelaron un riesgo considerable.

Se sugiere replicar el estudio con una muestra más amplia tanto en México como en otros países hispanoparlantes para contrastar la estructura factorial y las estimaciones reportadas. La validación cruzada en otros países con distinta lengua y cultura constituye para prueba de validez de constructo fuerte; no obstante, se puede revelar matices culturales que requieran su análisis y discusión ${ }^{17}$. Falta verificar la confiabilidad de la escala, sus factores e items a través de la estabilidad temporal; además, se pueden aportar pruebas de validez de constructo concurrente comprobando las correlaciones Journal Health NPEPS. 2018 jul-dez; 3(2):327-351. 
Valdez-Montero C, Rubia JM, Onofre-Rodríguez DJ, Benavides-Torres RA, Ahumada-Cortez JG. Validación...

positivas y moderadas o altas con búsqueda de sensaciones sexuales ${ }^{31}$ y conducta sexual compulsiva 32 .

\section{REFERENCIAS}

1. Organización Mundial de la Salud. VIH/sida, Datos y Cifras; 2018. Disponible en: http://www.who.int/es/news-room/fact-sheets/detail/hiv-aids

2. Centers for Disease Control and Prevention. HIV risk and prevention; 2016. Disponible en: http://www.cdc.gov/hiv/risk/index.html

3. Isacco A, Yallum N, Chromik L. A review of gay men's health: Challenges, strengths, and interventions. Am j lifestyle med. 2012; 1(6):45-62.

4. Organización Mundial de la Salud. Nuevos métodos de salud pública se dirigen a disminuir la propagación de la infección por el VIH y salvar vidas de hombres que tienen relaciones homosexuales y de transexuales; 2011. Disponible en: http://www.who.int/mediacentre/news/releases/2011/hiv_20110621/es/

5. Mustanski BS, Newcomb ME, Du Bois SN, Garcia SC, Grov C. HIV in young men who have sex with men: a review of epidemiology, risk and protective factors, and interventions. J sex res. 2011; 48(2-3):218-53. Doi:10.1080/00224499.2011.558645

6. Fernández $\mathrm{P}$, Zaragoza $\mathrm{K}$. Hombres jóvenes que tienen sexo con hombres: ¿un colectivo en alto riesgo para la infección por el VIH? Gac sanit. 2011; 25(5):372-8.

7. Phillips AE, Gomez GB, Boily MC, Garnett GP. A systematic review and meta-analysis of quantitative interviewing tools to investigate self-reported HIV and STI associated behaviours in low- and middle-income countries. Int J Epidemiol. 2010; 39(6):154155. Doi: $10.1093 /$ ije/dyq114.

8. Fendrich M, Smith EV Jr, Pollack LM, Mackesy-Amiti ME. Measuring sexual risk for HIV: a Rasch scaling approach. Arch Sex Behav. 2009; 38(6):922-35. Doi: 10.1007/s10508-008-9385-2.

9. Li J, Liu H, Liu H, Feng T, Cai Y. Psychometric assessment of HIV/STI sexual risk scale among MSM: a Rasch model approach. BMC Public Health. 2011; 11:763. Doi: 10.1186/1471-2458-11-763. 
Valdez-Montero C, Rubia JM, Onofre-Rodríguez DJ, Benavides-Torres RA, Ahumada-Cortez JG. Validación...

10. Newcomb ME, Ryan DT, Garofalo R, Mustanski B. The effects of sexual partnership and relationship characteristics on three sexual risk variables in young men who have sex with men. Arch Sex Behav. 2014; 43(1):61-72. Doi: 10.1007/s10508-013-0207-9.

11. Valdez, C. Modelo de conducta sexual en hombres que tienen sexo con hombre (Tesis de doctorado). Monterrey: Facultad de Enfermería, Universidad Autónoma de Nuevo León; 2015. 280 p.

12. Ato M, López JJ, Benavente A. Un sistema de clasificación de los diseños de investigación en psicología. Anal Psicol. 2013; 29(3):1038-59. Doi: 10.6018/analesps.29.3.178511.

13. Sáenz, N. E. Modelo para la promoción del sexo seguro en parejas estables (Tesis de doctorado). Monterrey: Facultad de Enfermería, Universidad Autónoma de Nuevo León; 2014. 260 p.

14. American Psychological Association. Ethical principles of psychologists and code of conduct. Washington: APA; 2017. Disponible en: http://www.apa.org/ethics/code/

15. Moorhead S, Johnson M, Maas ML, Swanson E. Nursing outcomes classification (NOC): measurement of health outcomes. 6th ed. St. Louis, MI: Elsevier/Mosby, 2018.

16. Lloret S, Ferreres A, Hernández A, Tomás I. El análisis factorial exploratorio de los ítems: una guía práctica, revisada y actualizada. Anal Psicol. 2014; 30(3):1151-69. Doi: 10.6018/analesps.30.3.199361

17. Byrne BM. Structural equations with AMOS: Basic concepts, applications, and programming. New York: Routledge; 2016.

18. Cho E, Kim S. Cronbach's coefficient alpha: Well-known but poorly understood. Organizational Research Methods. 2014; 18(2):207-30. Doi: $10.1177 / 1094428114555994$

19. Centro Nacional para la Prevención y Control del VIH y Sida. Vigilancia Epidemiológica de casos de VIH/SIDA en México. Registro Nacional de Casos de VIH y sida. 2018. Disponible en: https://www.gob.mx/censida/documentos/epidemiologiaregistro-nacional-de-casos-de-sida

20. ONUSIDA. Hoja informativa - Últimas estadísticas sobre el estado de la epidemia de sida. 2018. Disponible en: http://www.unaids.org/es/resources/fact-sheet 
Valdez-Montero C, Rubia JM, Onofre-Rodríguez DJ, Benavides-Torres RA, Ahumada-Cortez JG. Validación...

21. Hart GJ, Elford J. Sexual risk behaviour of men who have sex with men: Emerging patterns and new challenges. Curr opin infect dis. 2010; 23:39-44. Doi:10.1097/QCO.0b013e328334feb1

22. Velter A, Saboni L, Sommen C, Bernillon P, Bajos N, Semaille C. Sexual and prevention practices in men who have sex with men in the era of combination HIV prevention: Results from the Presse Gays et Lesbiennes survey, France. Euro surveill. 2015; 20(14):1-11.

23. Gaskin CJ, Happell B. On exploratory factor analysis: A review of recent evidence, an assessment of current practice, and recommendations for future use. Int $\mathrm{j}$ nurs stud. 2014; 51:511-21. Doi:10.1016/j.ijnurstu.2013.10.005

24. Weinstein N, Ryan WS, Dehaan CR, Przybylski AK, Legate N, Ryan RM. Parental autonomy support and discrepancies between implicit and explicit sexual identities: Dynamics of self-acceptance and defense. J pers soc psychol. 2012; 102(4):815-32. Doi:10.1037/a0026854.

25. Gondim RC, Kerr LR, Werneck GL, Macena RH, Pontes MK, Kendall C. Risky sexual practices among men who have sex with men in Northeast Brazil: results from four sequential surveys. Cad saúde pública. 2009; 25:1390-8. Doi:10.1590/\$0102$311 \times 2009000600021$.

26. Lyons A, Smith AM, Grierson JW, Von Doussa H. Australian men's sexual practices in saunas, sex clubs and other male sex on premises venues. Sex health. 2010; 7(2):18692. Doi: $10.1071 / \mathrm{SH} 09101$.

27. Valero S. Transformación e interpretación de las puntuaciones. En: Meneses J, editor. Psicometría. Barcelona: Editorial UOC; 2013.

28. Arreola S, Santos GM, Beck J, Sundararaj M, Wilson PA, Hebert P, et al. Sexual stigma, criminalization, investment, and access to HIV services among men who have sex with men worldwide. AIDS behav. 2015; 19:227-34. Doi: 10.1007/s10461-0140869-x.

29. Gutiérrez JP. Profile of gay men in Mexico City: results of a survey of meeting sites. Trop med int health. 2012; 17:353-60. Doi:10.1111/j.1365-3156.2011.02934.x

30. Meneses J. Aproximación histórica y conceptos básicos de la psicometría. En: Meneses J, editor. Psicometría. Barcelona: Editorial UOC; 2013. 
Valdez-Montero C, Rubia JM, Onofre-Rodríguez DJ, Benavides-Torres RA, Ahumada-Cortez JG. Validación...

31. Moral J. Validación de la Escala de Búsqueda de Sensaciones Sexuales en mexicanos casados o cohabitantes. Evaluar. 2018; 18(3): 49-72.

32. Scanavino M, Ventuneac A, Rendina HJ, Abdo CH, Tavares $\mathrm{H}$, Amaral ML et al. Sexual Compulsivity Scale, Compulsive Sexual Behavior Inventory, and Hypersexual Disorder Screening Inventory: Translation, adaptation, and validation for use in Brazil. Arch sex behav. 2016; 45(1): 207-17. Doi: 10.1007/s10508-014-0356-5

Conflito de interesses: Os autores declaram não haver conflito de interesses.

\section{Participação dos autores:}

- Concepção: Valdez-Montero C, Rubia JM, Onofre-Rodríguez DJ, BenavidesTorres RA, Ahumada-Cortez JG.

- Desenvolvimento: Valdez-Montero C, Rubia JM, Onofre-Rodríguez DJ, Benavides-Torres RA, Ahumada-Cortez JG.

- Redação e revisão: Valdez-Montero C, Rubia JM, Onofre-Rodríguez DJ, Benavides-Torres RA, Ahumada-Cortez JG.

Como citar este artigo: Valdez-Montero C, Rubia JM, Onofre-Rodríguez DJ, Benavides-Torres RA, Ahumada-Cortez JG. Validación de un inventario de conductas sexuales en hombres que tienen sexo con hombres. Journal Health NPEPS. 2018 jul-dez; 3(2): 327-351. 